\title{
Diagnóstico económico del criadero hacienda holstein Puculpala
}

\section{Economic diagnosis of the Holstein Puculpala farm}

\author{
Julio Cesar Benavides Lara. ${ }^{1}$, Tania Patricia Lara Cárdenas. ${ }^{2}$, Edwin Rafael Oleas \\ Carrillo. ${ }^{3} \&$ Monica Catalina Murillo Pinos. ${ }^{4}$
}

\section{DOI: https://doi.org/10.33262/visionariodigital.v4i3.1298}

\begin{abstract}
.
This project was created with the purpose of rescuing one of the livestock production activities of the Puculpala community of the Químiag parish belonging to the Riobamba Canton

The present study was carried out at the Holstein Puculpala Hatchery and aimed to obtain the gross and net margins of raw milk marketing; as well as identify the direct participation of the producer in the price of milk to the final consumer.

This work attempts to generate a contribution to facilitate decision-making at the financial level to ensure the permanence of the business, and to encourage farmers, the analysis of the cost so that it is a day-to-day element that allows continuous improvement in the process. Considering estimates, there is not enough information to adequately obtain the cost of production in the Hatchery.

The proposal of a costing system for the production of milk in the Holstein Holstein Puculpala Hatchery aims to determine the cost of production, the sale price and will achieve a better profitability by incorporating within the area of finance, a cost accounting unit, which It would basically have the following guidelines.

- Be a farm recognized by milk producers and marketers.

- Contribute to the development of the national industry looking for the product to be recognized for its quality, innovation and fair price.

- Have a national presence.

\footnotetext{
${ }^{1}$ Magister en Cadenas Productivas Agroindustriales, Ingeniero Zootecnista, Docente de la Escuela Superior Politécnica de Chimborazo, jbenavides@espoch.edu.ec

${ }^{2}$ Magister en Dirección y Gestión de Empresas de Servicio, Docente de la Escuela Superior Politécnica de Chimborazo, tania.lara@espoch.edu.ec

${ }^{3}$ Magister en Dirección de Empresas, Ingeniero Zootecnista, Docente de la Escuela Superior Politécnica de Chimborazo, eoleas@espoch.edu.ec

${ }^{4}$ Magister en Seguridad Industrial, Ingeniera Comercial, Gerente Comercial Agropecuaria Centro, mmurillopinos@hotmail.com
} 
- Be known for their practices and attitudes aligned with ethics and human values.

Keywords: Marketing, Producer, Costs.

\section{Resumen.}

Este proyecto nace con el propósito de rescatar una de las actividades productivas pecuarias de la comunidad Puculpala de la parroquia Químiag perteneciente al Cantón Riobamba.

El presente estudio se lo realizo en el Criadero Holstein Puculpala y se consideró como objetivos conseguir los márgenes brutos y netos de comercialización de la leche cruda; asi como determinar la participación directa del productor en lo que respecta al precio de la leche al consumidor final.

Este trabajo pretende generar una contribución para favorecer la toma de decisiones a nivel financiero para garantizar la permanencia del negocio y motivar a los ganaderos, el análisis del costo para que sea un elemento del día a día que perita el mejoramiento continuo en el proceso. Atendiendo estimaciones, no existe suficiente información para la debida obtención del costo de producción en el Criadero.

La propuesta de un sistema de costeo para la producción de leche en el Criadero Holstein Puculpala aspira determinar el costo de producción, el precio de venta y conseguirá una mejor rentabilidad incorporando dentro del área de finanzas, una unidad de contabilidad de costos, la cual comprenderá básicamente los siguientes lineamientos.

Ser una hacienda reconocida por los productores y comercializadores de leche.

Cooperar con el desarrollo de la industria nacional buscando que el producto sea reconocido por su calidad, innovación y precio justo.

Poseer presencia a nivel nacional.

Ser reconocida por sus prácticas y actitudes alineadas con ética y valores humanos.

Palabras clave: Comercialización, Productor, Costos.

\section{Introducción.}

En el Ecuador, en la Región Interandina, por sus condiciones medio ambientales ideales para producción de leche se reúne la mayor cantidad de hatos lecheros, de acuerdo a los datos del último Censo Nacional Agropecuario, el 76,79\% de producción nacional de leche se da en la Sierra, el 15,35\% en la región Costa y el 7,86\% en el Oriente y Región Insular (INEC, 2013).

La producción promedio de leche al día en el año 2013 según la Encuesta de Superficie y Producción Agropecuaria Continua (ESPAC) efectuada por el Instituto Nacional de Estadística y Censo (INEC, 2013) fue de 6.262.407 millones de litros de un total de 1.127.627 vacas ordeñadas (INEC, 2013).

Actualmente en el país hay 5,3 millones de cabezas de ganado bovino y 1,8 millones de ganado porcino, manifestó la Encuesta de Superficie y Producción Agropecuaria 
Continua (ESPAC) del Instituto Nacional de Estadística y Censos, cuyos resultados fueron difundidos por el Instituto Nacional de Estadísticas (Andes, 2012).

La industria láctea en el país está conformada por 40 compañías, las empresas se llevan casi la mitad de la producción lechera, la mayoría de haciendas ganaderas que producen leche están asentadas en la serranía ecuatoriana, donde se concentra el $75 \%$ de la producción nacional. El consumo promedio anual de un ecuatoriano al año es de 100 litros, aunque para los productores y según la OMS lo ideal son 150 litros de leche anuales (Diario el Telégrafo 2019).

La leche es un alimento indispensable en la dieta del ser humano, como fuente de proteína, grasa, vitaminas y minerales principalmente de calcio. Según estudios realizados por la Organización Mundial de la Salud y la Organización Panamericana de la Salud, un bebé de 6 a 23 meses de edad necesita consumir de 200 - 400ml de leche o yogur cada día, si es que consume productos de origen animal o $300-500 \mathrm{ml}$ de leche o yogur si no los consume (Organización Mundial de la Salud, 2010).

La leche de vaca es muy diferente a la leche humana, tanto en la cantidad como en la calidad de los nutrientes, es así que en lactantes menores de 6 meses no es recomendado el uso o sustitución de la leche de vaca por la leche materna, salvo casos excepcionales en los cuales puede ser modificada mediante la agregación de agua, azúcar y micronutrientes, con el objetivo de que pueda ser remplazada a corto plazo (Organización Mundial de la Salud, 2010).

En niños mayores a los 23 meses de edad época en que termina la lactancia materna, se aconseja dar de 1 a 2 vasos de leche de vaca diarios, como fuente de calcio para fortalecimiento del sistema óseo, crecimiento y aporte de proteínas, grasa y vitaminas (Organización Mundial de la Salud, 2010).

La leche y los productos lácteos tienen un gran potencial para mejorar la nutrición y los medios de subsistencia de cientos de millones de personas pobres en el mundo, según una nueva publicación de la FAO del 2013 en el libro "La Leche y los productos lácteos en la nutrición humana”, afirma que los gobiernos deberían invertir más en programas para hacer que la leche y los productos lácteos estén disponibles para las familias pobres y que les ayuden a producirlos en casa. "Como parte de una dieta equilibrada, la leche y los productos lácteos pueden ser una fuente muy importante de energía, proteína y grasas”, determinó la experta en nutrición de la FAO (Muehlhoff, 2013).

Muehlhoff recalcó que para contar con una dieta saludable es necesario una combinación de alimentos, la leche y productos lácteos no son las únicas fuentes de nutrientes esenciales, pero mientras la leche no se recomienda a niños menores de 12 meses, son un vehículo eficaz para conseguir nutrientes vitales y mejorar el crecimiento de los niños mayores a 12 meses, cuya nutrición es fundamental en los primeros mil días de vida, según la experta de la FAO (Muehlhoff, 2013). 
La composición de la leche puede verse influenciada por diversos factores genéticos y ambientales tales como: la raza, la edad, la etapa de lactación, el número de lactaciones, el nivel de producción, la estación del año, la dieta, la ocurrencia de enfermedades y otros muchos factores. El agua es el componente más abundante que hace que, el cloro, sodio y potasio estén en dispersión iónica, la lactosa y parte de la albumina en dispersión molecular, la caseína y fosfatos en dispersión coloidal y la materia grasa en emulsión (Giovambattista \& Peral, 2010).

\section{Metodología.}

n el Ecuador existen limitados estudios realizados en relación a la determinación o establecimiento de estructuras de costos para las unidades de producción agropecuarias dedicadas a la ganadería. Sin embargo, existen algunos trabajos de investigación que han sido manifestados principalmente por algunos institutos pertenecientes a universidades del país que por su ubicación geográfica se han visto en la necesidad de realizar investigaciones relacionadas con el tema tratado.

El presente análisis relacionado con los costos de producción del Criadero Holstein Puculpala, tiene como objetivo principal el de aportar y estandarizar el criterio sobre los costos existentes en las empresas pecuarias.

\section{Determinación del problema.}

El hato lechero del Criadero Holstein Puculpala, en la actualidad tiene un sistema de producción semi intensivo, la alimentación se basa en pastos como ray grass anual, ray grass perenne, trébol blanco, pero también refiere con una suplementación en vacas en producción a base de Silo de maíz, balanceado, y sales minerales. En el aspecto reproductivo, existen temporadas en que realizan la sincronización de celos en vaconas principalmente; las vacas y vaconas son servidas en su mayoría a través de inseminación artificial y cuando no se dispone de material genético con sometidas a monta natural, cuenta con pajuelas de toros Holstein americano y Holstein francés.

La ganadería del Criadero Holstein Puculpala en la actualidad carece de un control o seguimiento de todas las actividades de manejo productivo y reproductivo del Hato lechero. El problema principal es que no se cuenta con información necesaria de los parámetros productivos y reproductivos en condiciones de la serranía ecuatoriana.

Por lo anteriormente mencionado el presente estudio tiene como propósito señalar los puntos críticos del hato lechero del Criadero Holstein Puculpala, a través de la Evaluación Productiva y Reproductiva, basado en los escasos registros existentes para la toma de decisiones inteligentes en el manejo del hato que permitan mejoras en el aspecto productivo y reproductivo y conseguir una mejor rentabilidad estableciendo estrategias; teniendo en cuenta, que el ideal del técnico de la Área del presente estudio es la longevidad de los animales. 
ambién es necesario producir un sistema adecuado para comprender el costo de producción de un litro leche y así evitar posibles pérdidas en este tipo de producción y mejorar el rendimiento del Criadero Holstein Puculpala.

La razón es llegar a un establecimiento de los costos de producción con el objetivo de dar una orientación para que la información sea entendible.

Todas las empresas, sin importar el tamaño, necesitan entender el costo de producción bienes y servicios para poder llegar a fines propuestos y la toma de decisiones idóneas.

Este proyecto establecerá beneficios para los pequeños y grandes productores de leche ya que podrán analizar si la hacienda arroja ganancias o en su defecto pérdidas.

En la actualidad la explotación ganadera se ve afectada por los altos costos en alimentación e insumos, lo cual se puede observar en las tablas que se presentan a continuación.

Tabla 1. Comparación de precios en insumos de potreros

\begin{tabular}{ccccc}
\hline Insumo de potreros & Unidad & Año 2012 & Año 2013 & Año 2014 \\
\hline Trébol blanco & Libra & 2,11 & 3,20 & 3,50 \\
Trébol rojo & Libras & 3,15 & 2,20 & 2,40 \\
Ray grass anual & Quintal & 80,00 & 85,00 & 90,00 \\
Ray grass perenne & Quintal & 80,00 & 95,00 & 120,00 \\
Avena & Saco & 30,00 & 35,00 & 45,00 \\
Abono (Fertilización) & Quintal & 44,40 & 44,15 & 45,00 \\
TOTAL & & 239,66 & 264,55 & 305,90 \\
\hline
\end{tabular}

Fuente: Elaboración propia.

Figura 1. Comparación de precios en insumos de potreros

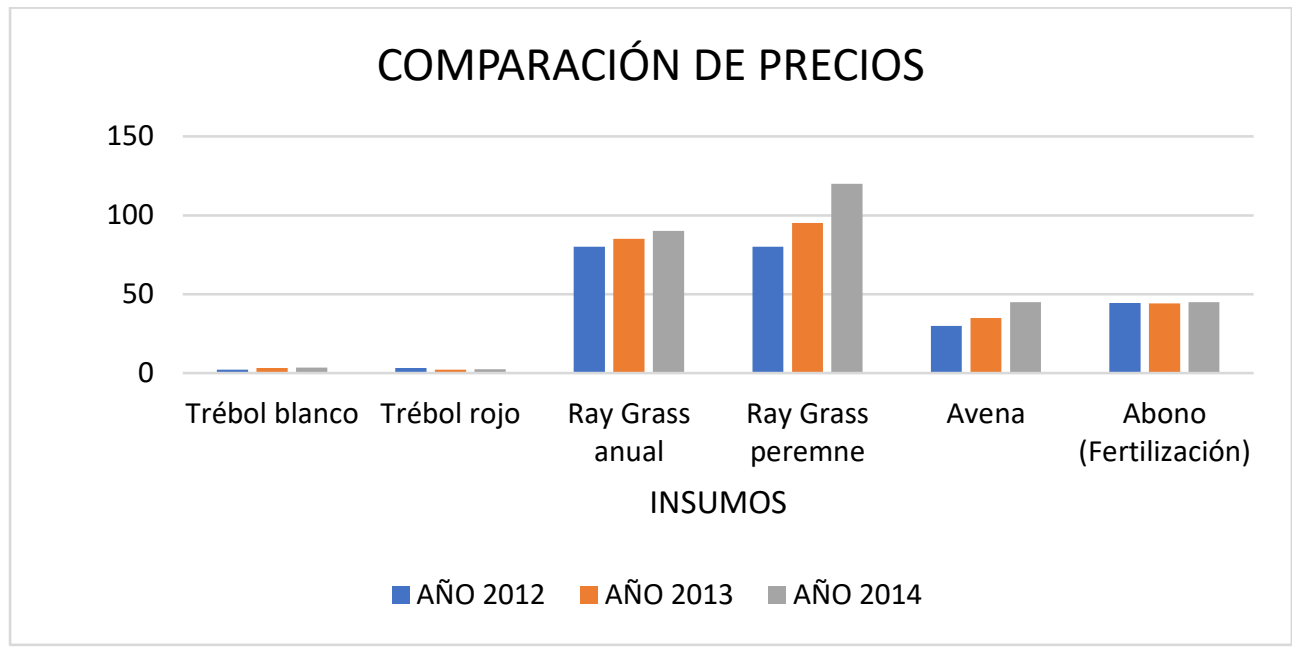

Fuente: Elaboración propia. 
Tabla 2. Variación de precios en insumos de potreros

\begin{tabular}{cccc}
\hline Insumos potreros & $\mathbf{2 0 1 2} \mathbf{- 2 0 1 3}$ & $\mathbf{2 0 1 3} \mathbf{- 2 0 1 4}$ & Variación \\
\hline Trébol blanco & 2,11 & 3,20 & $52 \%$ \\
Trébol rojo & 3,15 & 2,20 & $-30 \%$ \\
Ray Grass anual & 80,00 & 85,00 & $19 \%$ \\
Ray Grass perenne & 80,00 & 95,00 & $6 \%$ \\
Avena & 30,00 & 35,00 & $17 \%$ \\
Abono (Fertilización) & 44,40 & 44,15 & $-1 \%$ \\
\hline
\end{tabular}

Fuente: Elaboración propia.

Figura 2. Variación de precios en insumos de potreros

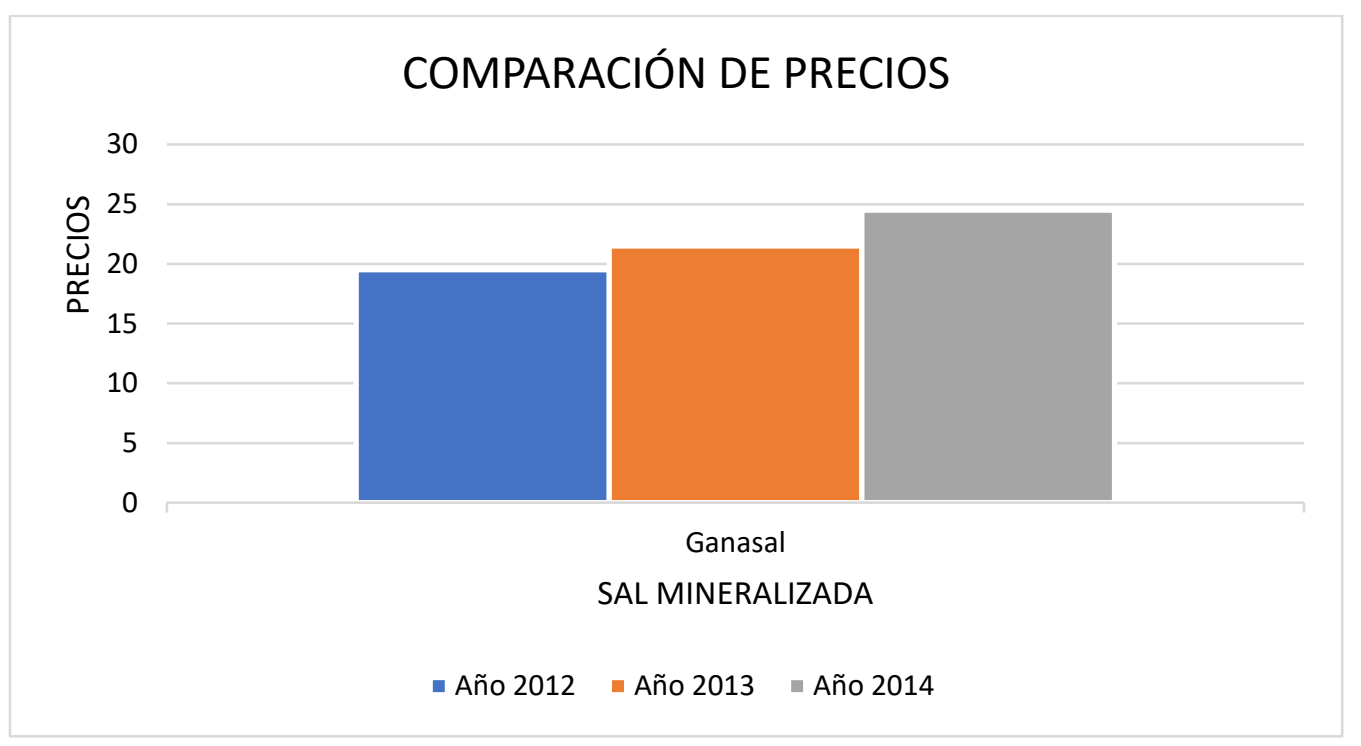

Fuente: Elaboración propia.

Claramente se puede observar la variación de precios de los principales insumos agrícolas para la elaboración de potreros que han tenido un alza significativa como es el trébol blanco, esta tuvo una variación de precios del $52 \%$; el trébol rojo tubo un decaimiento en el precio del $-30 \%$, de igual manera el fertilizante del $-1 \%$.

Tabla 3. Comparación de precios de la sal mineralizada

\begin{tabular}{cccc}
\hline Sal mineralizada & Año 2012 & Año 2013 & Año 2014 \\
\hline Ganasal & 19,50 & 21,50 & 24,50 \\
\hline
\end{tabular}

Fuente: Elaboración propia. 
Figura 3. Comparación de precios en sal mineralizada

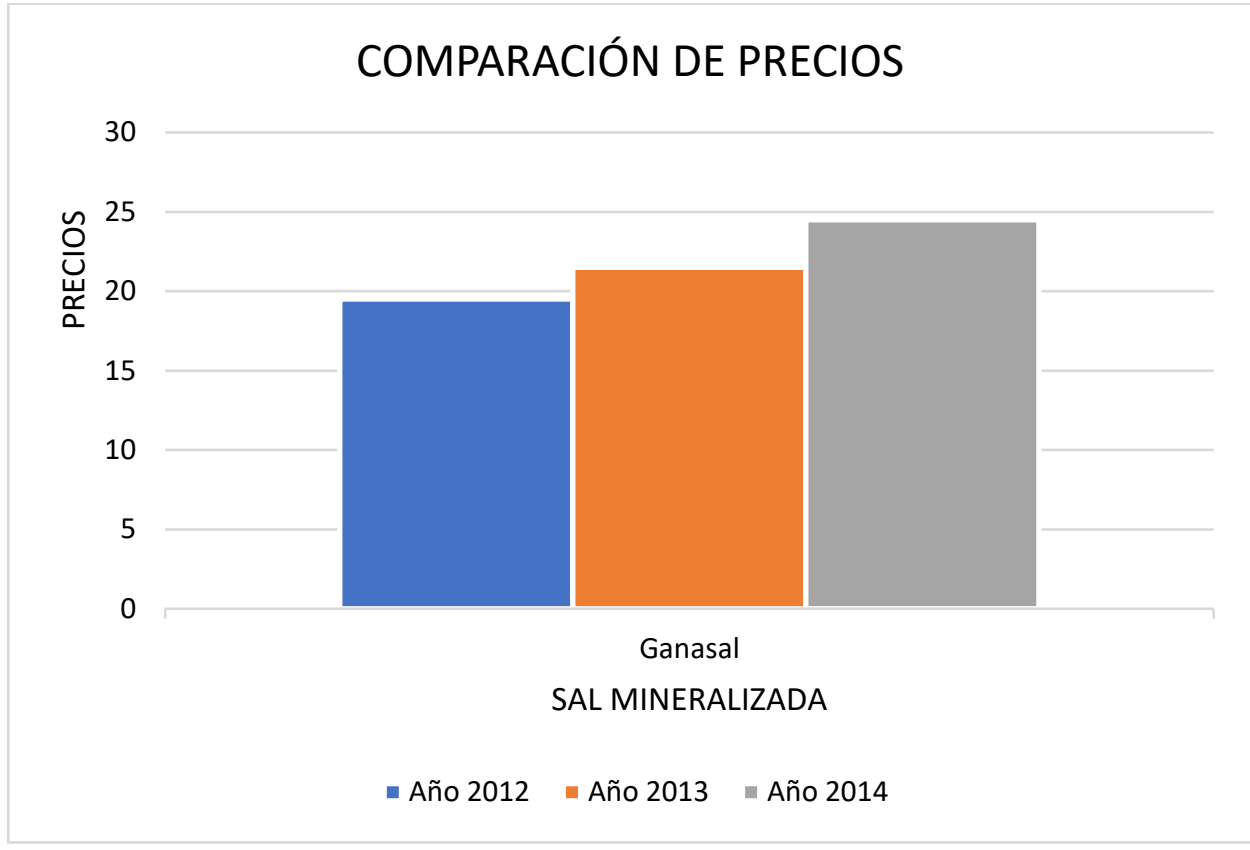

Fuente: Elaboración propia.

Tabla 4. Variación de precios de la sal mineralizada

\begin{tabular}{cccc}
\hline Sal mineralizada & $\mathbf{2 0 1 2 - \mathbf { 2 0 1 3 }}$ & $\mathbf{2 0 1 3 - \mathbf { 2 0 1 4 }}$ & Variación \\
\hline Ganasal (funda $20 \mathrm{Kg}$. ) & 21,50 & 24,50 & $14 \%$ \\
\hline
\end{tabular}

Fuente: Elaboración propia.

Figura 4. Variación de precios en sal mineralizada

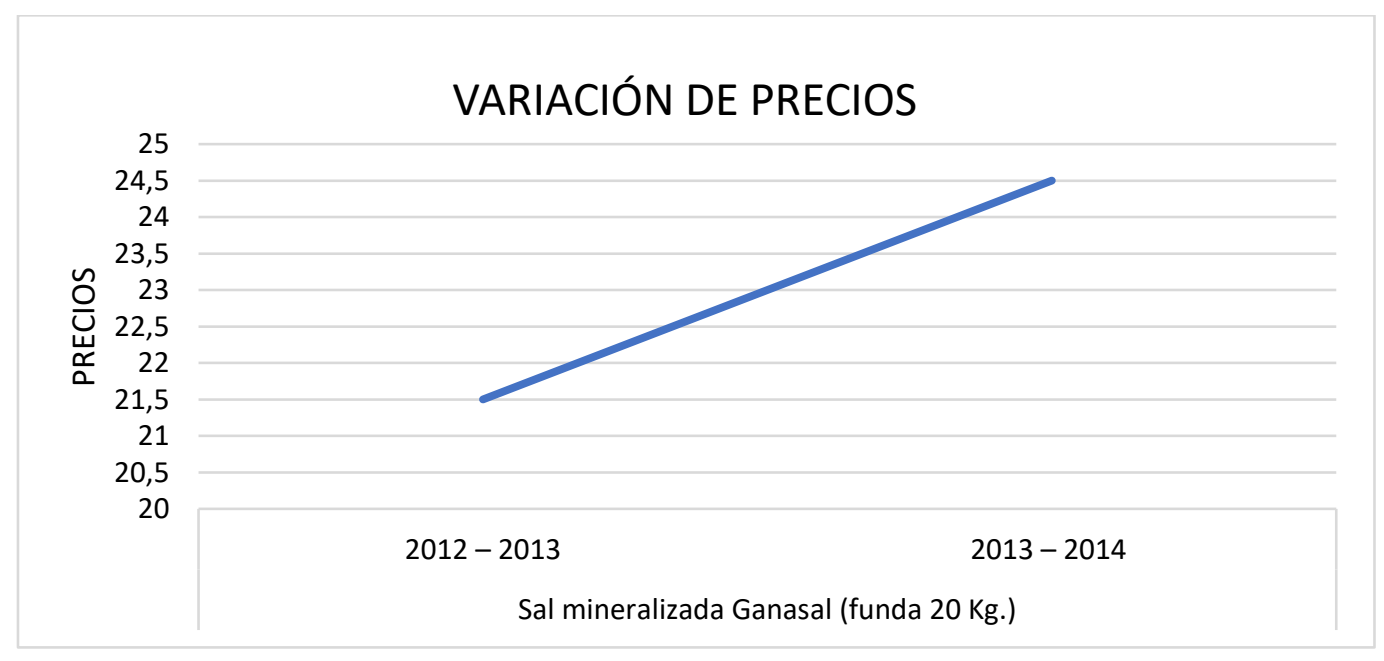

Fuente: Elaboración propia.

En la Tabla 6 se pude observa que los precios de la sal mineralizada de los últimos tres años han ido aumentando y como resultado tenemos una la variación del $14 \%$. 
Tabla 5. Variación de precios en medicamentos veterinarios

\begin{tabular}{cccc}
\hline Medicamentos & $\mathbf{2 0 1 2} \mathbf{- 2 0 1 3}$ & $\mathbf{2 0 1 3} \mathbf{- 2 0 1 4}$ & Variación \\
\hline Benzapen 20 ml. & 3,45 & 4,25 & $30 \%$ \\
Shotapent & 30,00 & 48,00 & $7 \%$ \\
Oxitócina 100 ml & 16,00 & 23,00 & $14 \%$ \\
Oxitetraciclina 50 ml. & 25,00 & 30,00 & $4 \%$ \\
Sorol 100 ml. & 9,00 & 13,00 & $29 \%$ \\
Desparasitantes 500 ml. & 18,00 & 20,00 & $20 \%$ \\
\hline
\end{tabular}

Fuente: Elaboración propia.

Figura 5. Variación de precios en medicamentos veterinarios

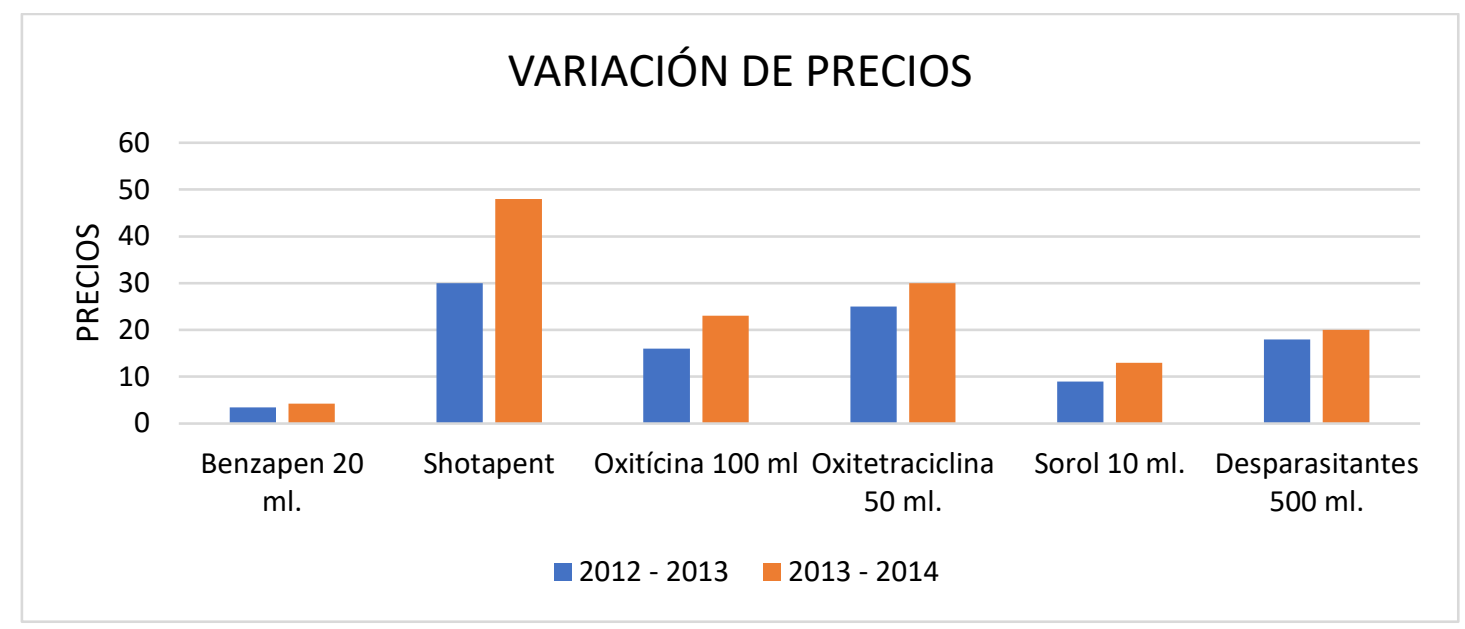

Fuente: Elaboración propia.

En la Tabla 7 se puede observar que la mayoría de los medicamentos de uso veterinario han tenido una elevada alza, como el Benzapen con una variación del $30 \%$ en los últimos tres años; de igual manera el Sorol con una variación del $20 \%$.

Tabla 6. Costos de producción y crianza de ganado vacuno

\begin{tabular}{|c|c|c|}
\hline Actividades & Valor & Porcentajes \\
\hline Mano de obra directa - producción & 471,61 & $15 \%$ \\
\hline Mano de obra indirecta & 51,13 & $2 \%$ \\
\hline Depreciaciones semovientes en crecimiento & 664,45 & $21 \%$ \\
\hline Materia prima (alimentación) & 1524,22 & $49 \%$ \\
\hline Materia prima (medicina y otros) & 109,90 & $4 \%$ \\
\hline Materiales indirectos & 19,90 & $1 \%$ \\
\hline Otros costos indirectos de fabricación & 81,78 & $3 \%$ \\
\hline Depreciación & 184,92 & 6 \\
\hline TOTAL & 3107,65 & $100 \%$ \\
\hline
\end{tabular}

Fuente: Elaboración propia. 
Figura 6. Costos de producción y crianza de ganado vacuno

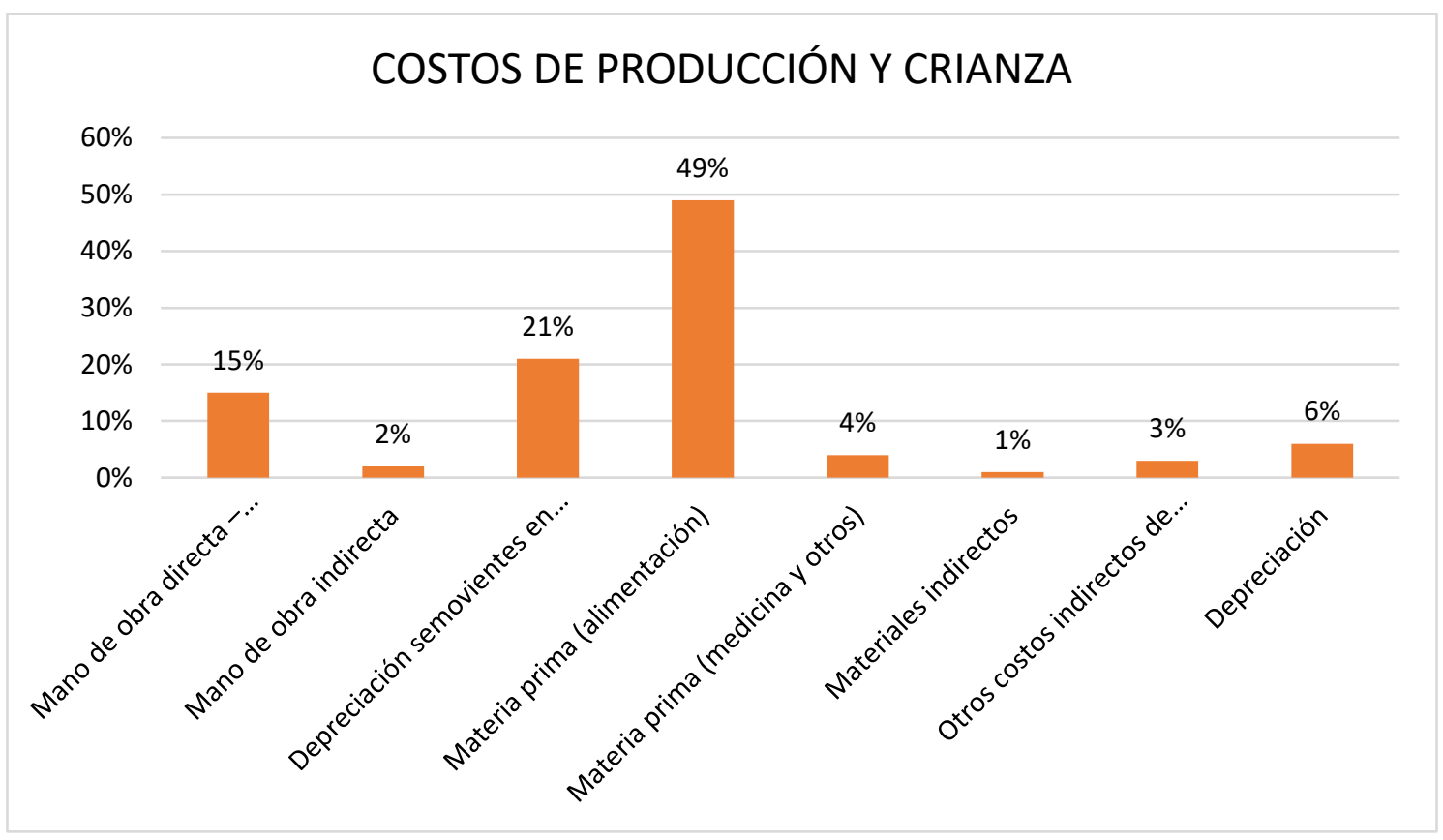

Fuente: Elaboración propia.

El propósito principal que lleva a realizar este trabajo investigativo es desarrollar un sistema de costos para estimar el costo de producción de un litro de leche en el Criadero Holstein Puculpala. El control de costos utilizado, es empírico, debido a que no se ha ejecutado una comprobación adecuada, lo que no ha permitido comprender el valor de producción, ni el margen de utilidad que genera o su pérdida.

\section{Sistematización del problema.}

¿Cómo afecta el desarrollo del sistema de costos y la medición de costo de producción en los resultados financieros en el Criadero Holstein Puculpala?

\section{Análisis situacional.}

Para realizar un estudio del sector agrícola- ganadero, es fundamental entender los factores que le afectan y favorecen, por lo cual se explicará el macro ambiente en el cual se desenvuelve.

Michel A, (1999). Es el mejor medio por el cual la empresa efectúa el reconocimiento de su realidad tanto en el ambiente interno como en el externo, y servirá para valorar, evaluar y analizar variables y factores tanto pasados, presentes, así como tendencia del futuro. 
Figura 7. Análisis situacional del sector agrícola ganadero

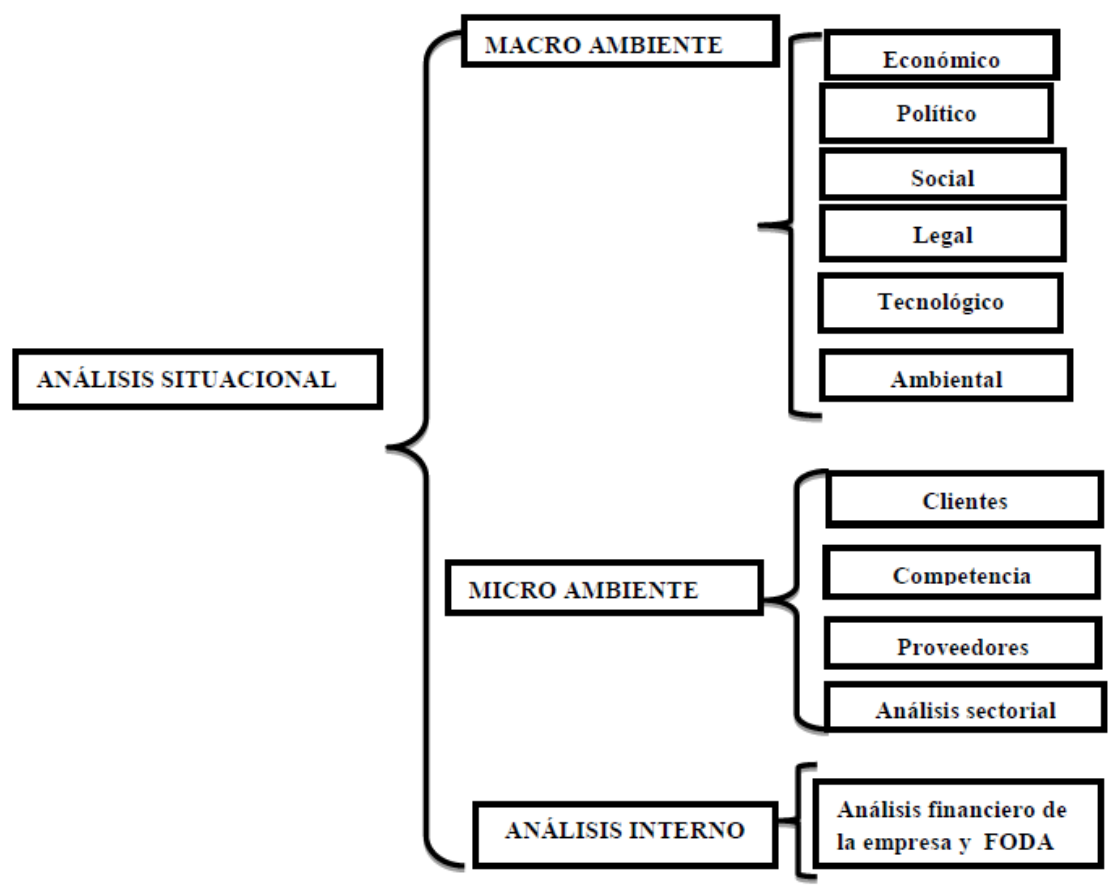

Fuente: Elaboración propia.

\section{Macro Ambiente.}

Mediante el análisis macro ambiental se establecerá las diferentes causas que influyen en el sector donde la empresa se encuentra, tales como, factores económicos, políticos, ambientales, tecnológicos que afectan el desempeño de las actividades y constituyen aspectos no controlables, como las oportunidades o amenazas para el Criadero Holstein Puculpala.

\section{Aspecto Económico.}

El Gobierno, a través del MAGAP (2011), "está encargado de coordinar, diseñar y evaluar las políticas del sector agropecuario con el resto de sectores económicos y sociales. Además, entre sus funciones está proveer condiciones de estabilidad y claridad en las reglas de juego que incentivan las inversiones privadas en el sector agropecuario.

El incentivo a las inversiones en esta área es importante para el desarrollo de la misma y a su vez produce nuevas fuentes de empleo. El efecto del ambiente económico sobre las empresas es enorme, pues determina el volumen de las operaciones, el nivel de precios y la rentabilidad potencial, la facilidad o dificultad de alcanzar los objetivos de las empresas, y el éxito o fracaso de estas estrategias.

La sociedad evolucionada muestra una diversidad en la economía, y el trabajo humano juega un papel fundamental. La actividad financiera se concreta en la producción de una 
amplia gama de bienes y servicios cuyo destino último es la satisfacción de las necesidades humanas.

Las actividades productivas de una sociedad se distribuyen a través de numerosas unidades productoras o empresas que individualmente emplean factores de producción como: trabajo, capital y recursos naturales para obtener bienes y servicios que después se ofrecen en el mercado. En el análisis económico, la diversidad de papeles que desempeñan los agentes económicos de un sistema productivo se puede ordenar las actividades en tres grandes sectores de producción:

- Sector primario. - Abarca las actividades que se ejecutan próximas a las bases de recursos naturales, esto es, la agrícola, pesca y ganadería.

- Sector secundario. - Recoge las actividades industriales, mediante las cuales los bienes son trasformados.

- Sector terciario o de servicios. - Reúne las actividades encaminadas a satisfacer necesidades de servicios productivos.

Larroulet C, Mochón, F, (2011). La macroeconomía se ocupa del estudio del funcionamiento de la economía en su conjunto. Su objetivo es obtener una visión simplificada de la economía pero que al mismo tiempo permita conocer y actuar sobre el nivel de actividad económica de un país determinado.

\section{Aspecto social.}

La población de la República del Ecuador en el año 2011 asciende a 14.889.500 habitantes, con la más alta densidad poblacional de América del Sur, con 56.5 habitante por Km2. La población está concentrada en su mayoría en el área urbana.

Tabla 8. Proyecciones de la Población por Área

\begin{tabular}{cccc}
\hline & Proyecciones de la Población por Área & \\
Año & Total & Urbana & Rural \\
\hline 2001 & 12156608,00 & 7431355,00 & 4725253,00 \\
2005 & 13798000,00 & 9093000,00 & 4705000,00 \\
2010 & 14483499,00 & 3600000,00 & 1500000,00 \\
\hline
\end{tabular}

Fuente: Elaboración propia. 
Figura 8. Proyecciones de la Población por Área

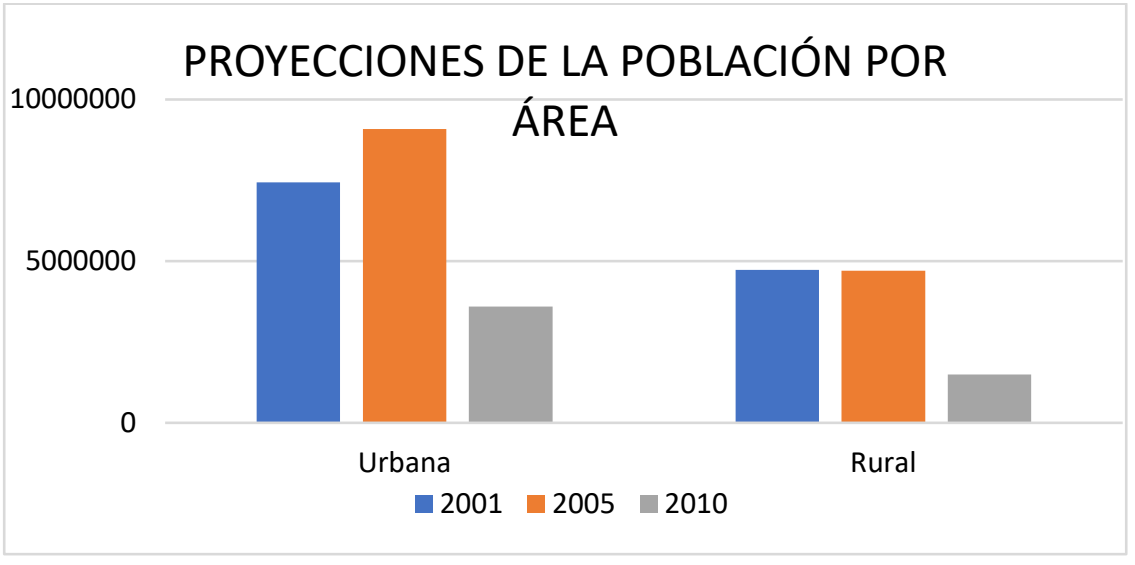

Fuente: Elaboración propia.

Según el último censo Nacional de Población y VI de vivienda 2010, la Población Económicamente Activa del Ecuador es de 4'585.575, de esta la actividad Agropecuaria ocupa el 26\% de la PEA (producción económicamente activa), siendo el 18\% ocupada por mujeres según indicadores en los cuadros. Se estima población económicamente activa a la población comprendida entre 12 y 65 años de edad que están cumpliendo o pueden cumplir funciones productivas.

Tabla 9. PEA por rama de actividad y por área urbana y rural

\begin{tabular}{|c|c|c|c|}
\hline \multicolumn{4}{|c|}{ PEA por rama de actividad y por área urbana y rural } \\
\hline Actividad & Total & Urbana & Rural \\
\hline $\begin{array}{l}\text { Agricultura, } \\
\text { ganadería }\end{array}$ & 4585575,00 & 2846004,00 & 1739571,00 \\
\hline Caza y silvicultura & 1203516,00 & 186649,00 & 1016867,00 \\
\hline
\end{tabular}

Fuente: Elaboración propia.

Figura 9. PEA por rama de actividad y por área urbana y rural

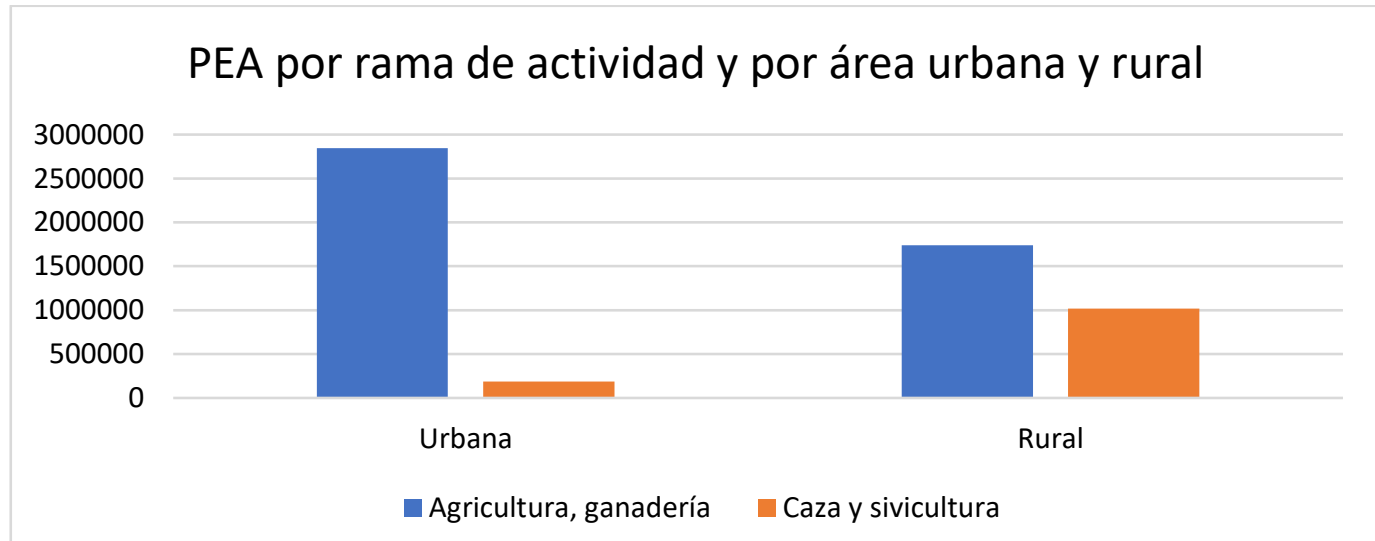

Fuente: Elaboración propia. 


\section{Aspecto político.}

El actual marco político agropecuario tiene como propósito el fortalecimiento de la institucionalidad del sector público y privado, el desarrollo de la agroindustria, mercados, sistemas de comercialización internos y externos, además entender la normativa y sistemas de sanidad e inocuidad agropecuaria, el financiamiento, inversión y uso de un seguro para el sector agropecuario, que permitirán la inserción en el mercados nacionales e internacionales; garantizando a su vez la sustentabilidad del rico patrimonio natural del país, procurando la igualdad de oportunidades para todos los agricultores, ganaderos y contacto con instituciones sólidas, trasparentes y eficientes públicas, privadas y gremiales que dan seguridad y confianza a los sectores productivos ecuatorianos.

A su vez se debe fomentar estrategias como la zonificación agraria, reactivación del sector público con visión de mercado junto a la priorización de cultivos, la diversificación de exportaciones, disminución de costos de producción, fortalecimiento institucional, recuperación de competencias del sector agropecuario y las provisiones de servicios complementarios como la investigación, capacitación, trasferencias de tecnología, riesgo, agroindustrial, créditos, comercialización entre otras para conseguir el desarrollo sostenible en el Ecuador con justicia social y libertad.

\section{Aspecto tecnológico.}

En el Ecuador, urgentemente se debe actualizar y reactivar el sector agropecuario, el MAGAP, en coordinación con instituciones y actores vinculados al sector, como las Cámaras de Agricultura, las Universidades, las Organizaciones no gubernamentales y los gremios han establecido el compromiso nacional de producir un sector agropecuario moderno con un desarrollo rural y territorial sostenible que garantice la seguridad alimentaria del país y su oferta exportable. En lo que respecta a lo tecnológico en el país a pesar que ha existido cambios importantes al compáralos con el de otros posiblemente estemos poco avanzados.

Si nos damos cuenta, en el país las actividades en las cuales la tecnología no ha dado mucho avance ha sido en el área agrícola ganadera, es por esto que la mayoría de las actividades agrarias se las realiza manualmente, y quedándose retrasados, en nuestra producción agrícola. Los únicos que han podido en algo implementar tecnología han sido los grandes empresarios.

MAG (2013) La producción ganadera en el Ecuador históricamente, ha facilitado el modelo de desarrollo, este ha sido básicamente de carácter extensivo, es decir, que el incremento de la producción se ha basado en la incorporación de más unidades de factores principalmente pastizales y número de cabezas de ganado, mas no, en mejoramiento de los rendimientos por unidades de factor, esto se demuestra en la baja producción de leche y carne. 


\section{Aspecto legal.}

En el Ecuador existen algunas instituciones que tienen relación con el sector agrícolaganadero del país, las mismas que aportan con información, asesoramiento técnico, ayuda económica a pequeños productores y agricultores y a las personas para que se aproveche la tierra en el cultivo.

MAGAP y sus instituciones integrantes

Esta Institución es un organismo del estado, que brinda información bibliográfica y se dedica también a prestar ayuda a los diversos proyectos, con respecto a direcciones con técnicos especializados en las diferentes áreas de la misma manera facilita fomento agropecuario a través de promotores.

AGROCALIDAD: Agencia Ecuatoriana de Aseguramiento de Calidad del Agro.

INCCA: Instituto Nacional de Capacitación Campesina

PROMSA: Programa de Modernización de Servicios Agropecuarios

Proyecto SICA: Servicio de Información y censo Agropecuario

Todos ellos dirigidos a facilitar asesoramiento y fortalecer las políticas institucionales y gubernamentales relacionados con el sector agropecuario.

- Instituto Nacional Ecuatoriano de Normalización

Que ha establecido y propagado las normas relacionadas con la leche y sus derivados, en ellas se establecen los diversos requisitos que debe cumplir la leche en su estado natural o crudo, pasteurizado y en polvo.

- Instituto Nacional de Investigación Agropecuarias (INIAP)

Esta institución cuenta con personal capacitado para ofrecer información, de asesoramiento técnico sobre productos agrícolas y a la vez brinda apoyo técnico para la realización de ciertos proyectos relacionados con la agricultura. Cabe recalcar que la institución posee en diferentes poblaciones del país granjas experimentales, en las cuales algunos productos son puestos a prueba.

- Corporación Financiera Nacional (CFN)

La CFN es una institución pública, autónoma, con personaría jurídica, técnica, moderna, ágil y eficiente que, incita la modernización y el crecimiento de todos los sectores productivos y en servicio, coadyuvando una nueva cultura empresarial, orientada a lograr mejores niveles de eficiencia y competitividad. 
Es la primera institución financiera en Ecuador en tener el Certificado de calidad ISO 9001.

Siguiendo su estrategia de banca de desarrollo, la Corporación Financiera Nacional dispone de facilidades crediticias y productos financieros destinados al financiamiento de los sectores productivos y de servicios del Ecuador.

Adicionalmente, la CFN brinda servicios de capacitación y asistencia técnica a través de los Centros de Apoyo Empresarial en procura de una nueva cultura financiera y empresarial que privilegie la inversión productiva.

En el sector agrícola ganadero la CFN no ha tenido una buena cobertura en el sector rural, eso se debe a la falta de una publicidad por parte de la Institución.

\section{- Banco Nacional de Fomento (BNF)}

El BNF es una institución pública que promueve el desarrollo socio-económico y sostenible del país con equidad territorial, enfocado principalmente en los micro, pequeños y medianos productores a través de servicios y productos financieros al alcance de la población.

Esta institución financiera pública que brinda apoyo a través de diversas líneas de crédito para la producción con bajas tasas de interés para el sector este financiamiento ayuda a los productores a seguir con sus producciones y a mejorar su infraestructura.

\section{Micro ambiente.}

Por medio del microambiente se realizará una identificación de los elementos con los cuales la entidad procede de forma directa durante la ejecución de sus actividades los mismos que no pueden ser controlados por la empresa; entre estos se encuentra los siguientes: los clientes, proveedores y competidores.

\section{Clientes.}

Son personas, entidades u organizaciones que compran un producto o servicio para cubrir un requerimiento o necesidad. Cualquier negocio depende de la cantidad de clientes que dispone para mantener su posición en el mercado y orientará sus capacidades, recursos y destrezas para obtener sus elecciones o preferencias.

La misión de toda empresa debe ser de mayor nivel de satisfacción para sus clientes.

El cliente principal, el cual compra el cien por ciento de la producción, es el señor Segundo Asintinbay, el cual se destina a la compra de leche cruda en las haciendas del sector, su objetivo es de procesar la leche en forma artesanal y de sacar sus derivados para ofrecer al mercado. 


\section{Proveedores.}

Un proveedor puede ser una persona o entidad que abastece de insumos a otra empresa.

El Criadero Holstein Puculpala tiene varios proveedores de insumos y materiales agrícolas ganadero y de mano de obra de obra (Veterinario), estos deben cumplir con las exigencias del mercado teniendo productos y servicios de calidad.

Tabla 10. Proveedores de insumos

\begin{tabular}{cc}
\hline Proveedor & Insumos \\
\hline Agropecuaria Centro & Medicamentos y desinfectantes de uso veterinario \\
Agro-Orgánicos & Insumos agrícolas \\
Inexagro & Semen bovino (inseminación artificial) y \\
Fertisa & nitrógeno. \\
\hline
\end{tabular}

Fuente: Elaboración propia.

\section{Competidores.}

Se considera competidores a un conjunto de empresas que coinciden en un mismo mercado y ofrecen productos similares.

Los competidores forman las haciendas aledañas del sector, que se dedican a la producción de leche cruda, compiten en calidad para entregar el producto a las plantas procesadoras sean estas a nivel industrial o artesanal, éstas deben cumplir con los siguientes estándares de calidad o requisitos. A mejor calidad mejor precio por litro de leche.

Diagnóstico del sector pecuario de la comunidad puculpala - parroquia químiag cantón Riobamba.

\section{Georreferenciación.}

Figura 10. Ubicación Geográfica

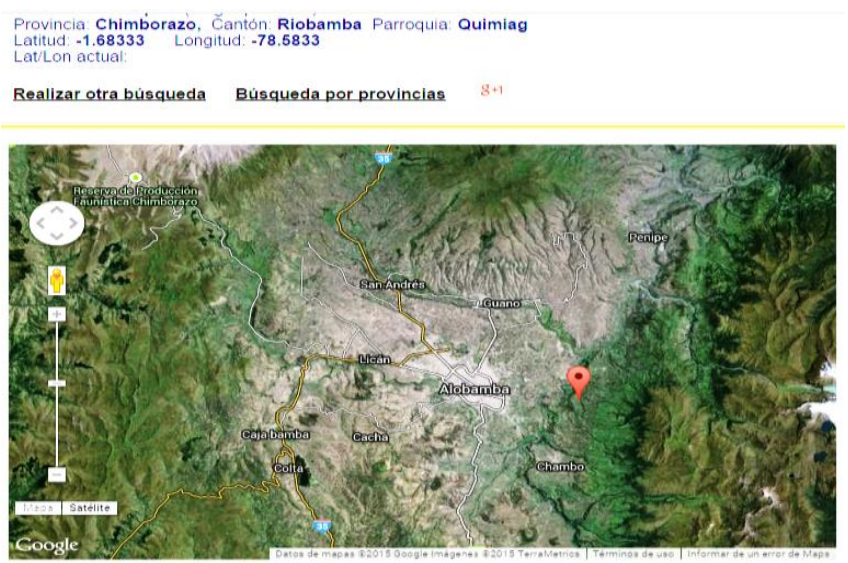


Fuente: Google Maps

\section{Análisis FODA del sector ganadero}

Tabla 11. Análisis FODA del sector ganadero

\begin{tabular}{|c|c|}
\hline \multicolumn{2}{|c|}{ ANÁLISIS FODA } \\
\hline $\begin{array}{l}\text { Fortalezas } \\
\text { - El aumento del PIB permitirá el } \\
\text { crecimiento de circulación dando } \\
\text { mayor oportunidad de compra. } \\
\text { - Sector altamente activo para la } \\
\text { soberanía alimenticia. }\end{array}$ & $\begin{array}{l}\text { Oportunidades } \\
\text { - Las carreteras en buen estado ayudan a } \\
\text { trasportar la producción a las grandes } \\
\text { ciudades. } \\
\text { - Desarrollo de la agroindustria para dar un } \\
\text { valor agregado a los productos. } \\
\text { - Competitividad en calidad y costo del } \\
\text { producto. }\end{array}$ \\
\hline $\begin{array}{l}\text { Debilidades } \\
\text { - Crisis de productividad lechera y } \\
\text { en el sector de carne. } \\
\text { - El costo de producción de leche } \\
\text { caro. } \\
\text { - Bajo nivel de educación de los } \\
\text { productores lecheros. } \\
\text { - Sector con alto potencial } \\
\text { productivo, pero con una canasta } \\
\text { básica cara }\end{array}$ & $\begin{array}{l}\text { Amenazas } \\
\text { - } \text { Poca prioridad e insuficiente inversión por } \\
\text { parte del gobierno para el sector agrícola } \\
\text { ganadero. } \\
\text { - Falta de políticas para el desarrollo del } \\
\text { sector ganadero. } \\
\text { - El elevado costo de los insumos es un } \\
\text { factor externo que impacta directamente a } \\
\text { la rentabilidad de los productores. } \\
\text { - Corporación Financiera y el Banco de } \\
\text { Fomento ayudan con capital de trabajo, } \\
\text { pero hipotecan las propiedades con el } \\
\text { riesgo de perder las tierras. } \\
\text { - Poco apoyo financiero para el sector } \\
\text { ganadero. } \\
\text { - Mano de obra cara. } \\
\text { Créditos con altas tasas de interés. }\end{array}$ \\
\hline
\end{tabular}

Fuente: Elaboración propia.

\section{Análisis interno.}

Análisis financiero del Criadero Holstein Puculpala y análisis FODA

Es un conjunto de técnicas y procedimientos de análisis cuyo propósito final será el de interpretar de manera lógica y ordenada las diferentes cuentas y grupo de cuentas de los estados financieros, de tal manera se verificará la capacidad financiera de la empresa.

\section{Objetivos del análisis financiero:}

- Medición y desempeño que ha tenido la hacienda en los últimos tres años.

- Medir los indicadores de liquidez. 
- Medicar la capacidad financiera de la empresa.

Tabla 12. Análisis FODA del Criadero Holstein Puculpala

\section{ANÁLISIS FODA}

\section{Fortalezas}

- Recursos propios.

- Infraestructura en buen estado.

- Buena administración

- Personal capacitado.

- Bajos costos de producción ganadera.

- Buen manejo del hato ganadero en sanidad.

- Mejor precio por litro de leche.

\section{Debilidades}

- Falta de un sistema de costos.

- Falta de normas procedimientos Contables.

- No contar con el apoyo de otras empresas para el desarrollo particular.

- Falta de empresas aseguradoras para el sector ganadero.

\section{Oportunidades}

- Producción de buena calidad en el sector ganadero de la zona.

- Buen manejo económico de las haciendas líderes del sector lechero.

- Una buena estrategia económica para producción.

- Saber manejar las tierras a costo de capital propio, y buscar un valor agregado a la producción.

Fuente: Investigación directa en el Criadero Holstein Puculpala.

\section{Conclusiones:}

- La

\section{Referencias bibliográficas:}

Giovanbattista, G. - Peral, P Genética de Animales Domésticos, Intermedica (2010) México: Mc Graw Hill

Cristián Larroulet - Francisco Mochón, Economía, Editorial Mc Graw Hill (2003)

Michel A, (1999). Administración Estratégica: Thomson Editores.

Ministerio de Agricultura, Ganadería y Pesca (2011). Funciones del Ministerio.

Corporación Financiera Nacional (2009). Información Corporativa. 
Banco Nacional de Fomentos. (2014). Información Corporativa.

Glosario Marketing Online (2012). Definición de Competencia http://www.innoconsulting.com.ar/html/pdf/inno/glosario.pdf

Diario la hora.En:http://www.lahora.com.ec/ind dos tema sex.php/noticias/show/1101431216/

Instituto Nacional de Estadísticas y Censos INEC. (2006/2015). Análisis y Proyecciones de la Población Económicamente Activa (PEAD) del Ecuador.

Ministerio de Agricultura y Ganadería. (MAG). Consultado:12 de mayo del 2013.

Instituto Nacional de Estadísticas y Censos (INEC), Encuesta de Superficie y Producción Agropecuaria Continua - ESPAC https://www.ecuadorencifras.gob.ec/encuestade-superficie-y-produccion-agropecuaria-continua-bbd/

Instituto Nacional de Estadísticas y Censos (INEC), Anuario estadístico 2012 https://www.ecuadorencifras.gob.ec/inec-presenta-resumen-estadistico-2012/

Diario El telégrafo. https://www.eltelegrafo.com.ec/noticias/economia/4/bajo-consumolacteos-ecuador

Principios de orientación para la alimentación de niños no amamantados entre los 6 y los 24 meses de edad, Departamento de Salud y Desarrollo del Niño y Adolescente Organización Mundial de la Salud, http://www.who.int/child-adolescent-health

\section{\Ciencia}




\section{PARA CITAR EL ARTÍCULO INDEXADO.}

Benavides Lara, J. C., Lara Cárdenas, T. P., Oleas Carrillo, E. R., \& Murillo Pinos, M. C. (2020). Diagnóstico económico del criadero hacienda holstein Puculpala. Visionario Digital, 4(3), 102-121. https://doi.org/10.33262/visionariodigital.v4i3.1298

\section{LCiencia}

El artículo que se publica es de exclusiva responsabilidad de los autores y no necesariamente reflejan el pensamiento de la Revista Visionario Digital.

El artículo queda en propiedad de la revista y, por tanto, su publicación parcial y/o total en otro medio tiene que ser autorizado por el director de la Revista Visionario Digital.
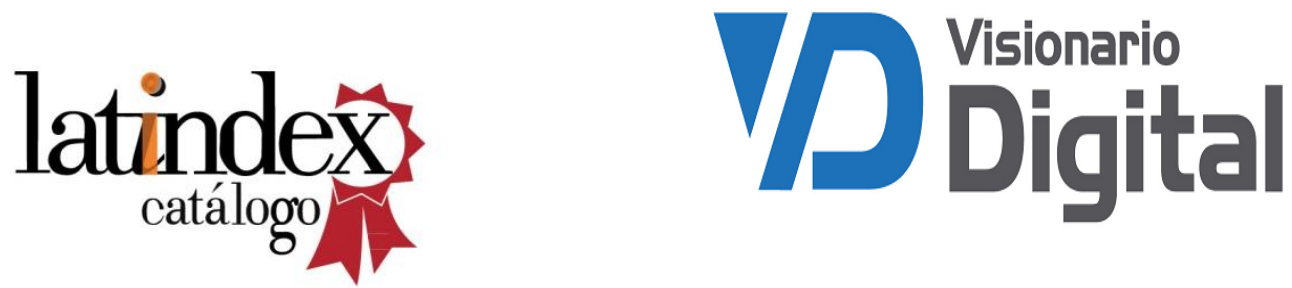